\title{
USING AN INERTIAL NAVIGATION SYSTEM FOR GRAVIMETRIC APPLICATIONS. A COMPARATIVE STUDY BETWEen AN INS, A MICROGRAVITY METER AND A SEISMOMETER.
}

\author{
Benjamin Beirens, José Darrozes, Guillaume Ramillien and Lucia Seoane \\ Géosciences Environnement Toulouse, Benjamin.Beirens, Guillaume.Ramillien, José.Darrozes, \\ Lucia.Seoane@get.omp.eu
}

\begin{abstract}
The goal of this study is to test the feasibility of gravimetric measurements with a small Inertial Navigation System (INS) unit in order to use it with various platforms (drone, plane, satellite) in the future. The INS operated in this study is the SPATIAL model developed by Advanced Navigation which has 3-axis accelerometers of precision $2.10^{-4} \mathrm{~m} / \mathrm{s}^{2}$. To start we shall compare its gravimetric capabilities with an LPS-1 seismometer (Micro-g LaCoste) during an in situ experiment over a long period of time at the Géosciences Environnement Toulouse (GET) laboratory in order to see if the INS can detect the varying gravimetric signals at least related to the Earth tides. Then we'll compare its precision with the measurements made by a CG-5 Autograv gravimeter (Scintrex) during an excursion between the GET laboratory and the Cammazes dam, which has an altitude difference of more than 400 meters so the gravity variation should be detectable.
\end{abstract}

Index Terms - INS, accelerometers, gravimetry

\section{STATE-OF-ART}

Studies have been done with large, robust inertial navigation systems [1][2]. However due to their size and weight the applications of these acquisition systems are limited to large UAV vehicles. We propose a similar study that was done with a much smaller inertial navigation system, which will allow for the possibility of being installed in smaller platforms like drones.

\section{MATERIALS}

The INS SPATIAL model developed by Advanced Navigation we used is a miniature INS ( $3 \mathrm{~cm} \times 4 \mathrm{~cm} \times 2 \mathrm{~cm})$ equipped with 3 -axis accelerometers (precision $2.10^{-4} \mathrm{~m} / \mathrm{s}^{2}$ ), gyroscopes (precision $0.2^{\circ}$ ), magnetometers, a thermometer, a barometer and a GNSS module (precision $2.0 \mathrm{~m}$ ).

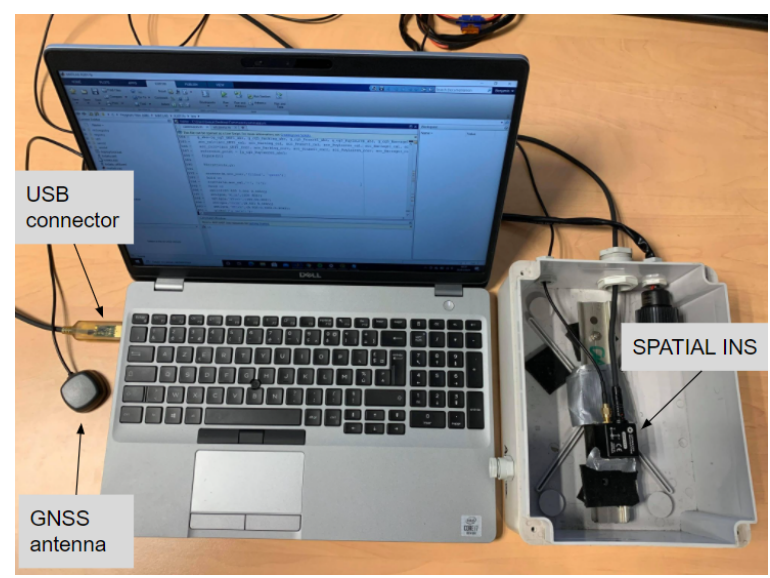

Figure 1: The SPATIAL INS attached in a box that can be closed to reduce influence of outside temperature fluctuations. The INS is connected to a GNSS module and a PC using USB.

\section{THE DIFFERENT EXPERIMENTS}

During a two-month period (July-September 2020) the inertial navigation system and seismometer were placed in the same location at the GET laboratory. There were two main goals for this in situ experiment in static conditions. The first was to compare the gravity measurements of the INS with those of a LPS-1 seismometer which is a very precise but bulky instrument and find out if the INS measurements were precise enough to detect the signals related to the Earth tides. The second goal was to study the influence of different factors, in particular warming-up temperature, on the precision of the INS measurements in order to find the best setting for future experiments.

The next step was to use the INS unit at different measurement sites but in static mode. On the 18th of November, 2020 an excursion was done from the GET laboratory (lon: $1^{\circ} 28^{\prime} 41.6^{\prime \prime E}$ lat: $43^{\circ} 33^{\prime} 41.3^{\prime \prime} \mathrm{N}$ ) to the Cammazes dam (lon: 25'39.1'E lat: 43²4'26.8”N) and back. The goal of this experiment was to find out if such an INS acquisition system could be used to detect the gravity anomaly due to the altitude difference between the two measurement sites which is 421 meters. The measurement 
values recorded by the SPATIAL INS unit were made every minute continuously during the whole day. Multiple stops were made on the way in order to measure the gravity using the relative CG-5 gravimeter used as reference points. The gravimeter made one measurement every minute and in total three measurements were made at every stop. If the recorded values didn't deviate more than $+/-0.05$ mGal the measurements were kept, if not a new acquisition of three measurements would be done. Since we didn't stay for the same duration at each location, there would be a different amount of measurements made by the SPATIAL INS at each stop.

Because the measurements made by the gravimeter are relative measurements, at least one stop had to be made at a reference point. Our reference point was at Puylaurens (reference system RGF93 lon: $2^{\circ} 00^{\prime} 14.86098^{\prime \prime} \mathrm{E}$, lat: $43^{\circ} 34^{\prime} 04.63240$ 'N height: $340.303 \mathrm{~m}$ ). At this location the absolute gravity measured by IGN in 2019 was 980396.906 $\mathrm{mGal}$ with a precision of $20 \mu \mathrm{Gal}$. From this reference point we can deduce the value of the absolute gravity at the other locations by adding the difference of the measured relative value between each location and this reference point at Puylaurens.

\section{RESULTS}

From our in situ experiment we learned that having a too high sampling rate $(10-100 \mathrm{~Hz})$ yields very noisy measurements. Because the main goal of this experiment was to see if the SPATIAL INS unit is precise enough to measure absolute gravity and possibly its varying part, e.g. the solid Earth tides, having a sampling of 60 seconds for both the INS and seismometer would be more than enough. We also noticed a deviation of the accelerometer measurements which was correlated to the temperature provided by the instrument. This means that if the SPATIAL INS unit starts warming up during an acquisition, which would happen after only a few minutes, a correction would be required.

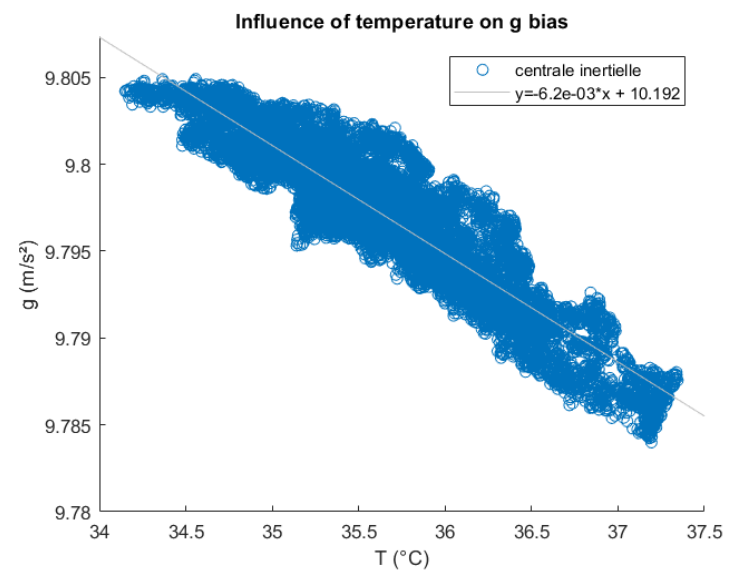

Figure 2: Gravity data $\left(\mathrm{m} / \mathrm{s}^{2}\right)$ plotted against temperature of the INS showing a linear correlation (data from 03/09/2020-15/09/2020 at $0.0166 \mathrm{~Hz}$ ).
Using Tsoft [4] we obtained the gravimetric signals related to the Earth tides during the lapse of time of our experiment at the GET laboratory. The cross wavelet transform of our gravity measurements reveals a strong correlation for the daily and sub-daily periods of 12-24 hours. This corresponds to the fundamental periods of the solid Earth tides, meaning the gravity data of the SPATIAL INS is indeed precise enough to detect them.

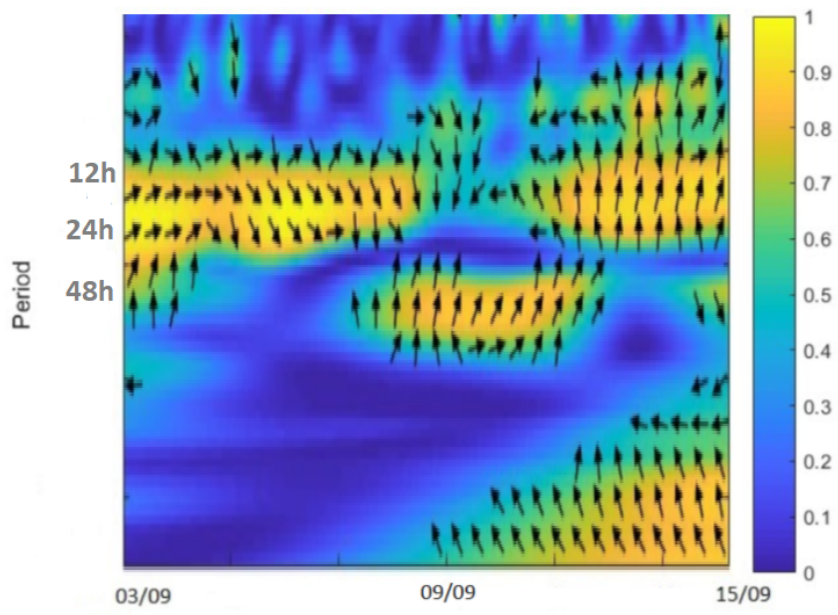

Figure 3: Wavelet coherence of our INS gravity data and Solid tide data for the period of 03/09/2020-15/09/2020.

During our campaign to the Cammazes dam, in order to measure the instrumental deviation of the CG-5 gravimeter throughout the day, relative gravity measurements were made at three locations twice during the day, e.g. the total deviation between the starting and end moment at the GET laboratory was $0.1 \mathrm{mGal}$ over an 11-hour period. To correct this bias at each location we assume this deviation to progress linearly throughout the acquisition period.

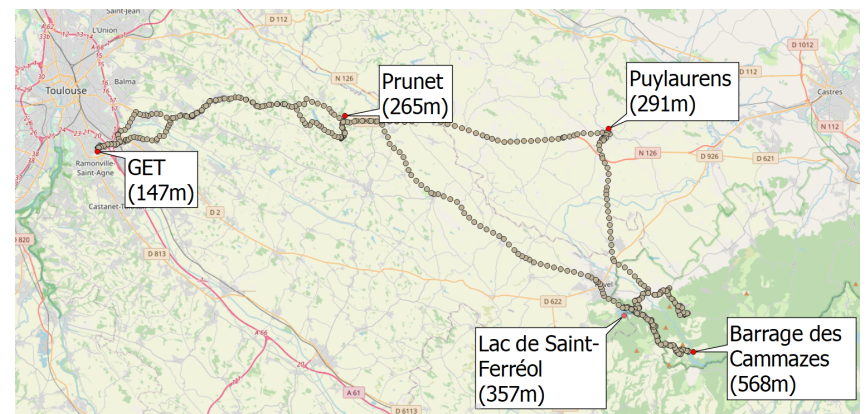

Figure 4: The GPS data from our campaign, with the height of different locations added.

For future applications it is possible to derive the gravity on a mobile vehicle by considering the difference between the INS measure of the specific forces and the kinematic acceleration deduced from the GNSS positioning, by applying Newton's second law of motion in the inertial frame. [4]

$$
g^{i}=a^{i}-\ddot{X}^{i}
$$


Depending on the time we stayed at each site, a different amount of INS unit samples were considered to compute an average gravity. Because we could not ensure the INS to be perfectly level, we calculated the norm of the acceleration vector for each measurement axis, instead of considering only its given z-axis component that would not have the exact vertical direction, so that the norm of the gravity vector can be plotted for every stop of the experiment. As it is necessary for the gravimeter, the SPATIAL INS unit needs to be calibrated, e.g. by using the measurements made at the GET laboratory (starting point) as a reference gravity point as we had already collected a lot of data for this place.

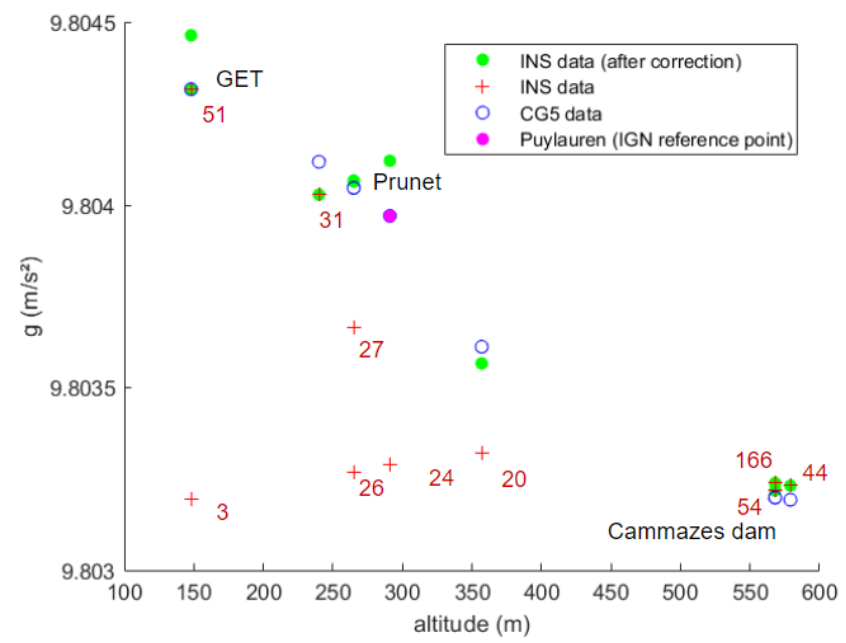

Figure 5: Gravity data $\left(\mathrm{m} / \mathrm{s}^{2}\right)$ versus altitude given by the INS (before and after correction) with the number of measurements for each stop, CG5 gravimeter with the IGN reference point.

When we plot the INS data before any correction (red crosses), as seen on Figure 3, it is clear that it barely matches up with the precise measurements made by the more accurate CG-5 gravimeter (blue circles). We notice that the stops of less than 30 measurements give very poor results, with differences of more than $5.10^{-4} \mathrm{~m} / \mathrm{s}^{2}$, but on the other hand the locations where we stopped for a longer period of more than 30 minutes, the gravity values from the INS unit are closer to those obtained by the CG-5 gravimeter. Because we made two stops at the GET laboratory, Prunet and the Cammazes dam, the difference in accelerometric data and temperature for the first and second visits at each of these sites give information on their dependency. In particular, the deviation in gravity measurements appears to be linearly correlated to the difference in temperature, as it was suspected during our first in situ experiment. A detrending correction is thus applied to each INS gravity value that was obtained by averaging less than 30 measurements which showed an important difference with the reference CG- 5 gravity values.

\section{PERSPECTIVES AND CONCLUSIONS}

Even though our first in situ experiment showed us that the SPATIAL INS unit's performance is good enough to detect the Earth tides, we plan on doing another in situ experiment, where we will eliminate and investigate other factors that influence the performance of this INS unit (a room with less temperature fluctuation, comparing data of multiple SPATIAL INS measuring at the same time but with different frequencies, adding the CG5-gravimeter data).

Once they are corrected from the temperature effects and sufficiently averaged versus time, the INS gravity measurements made in the field show very promising results and offer new perspectives for gravity measurements on a vehicle such as a drone, once they are combined with kinematic trajectories.

\section{REFERENCES}

[1] C. Lin, K. Chiang, and C. Kuo, "Development of INS/GNSS UA-Borne Vector Gravimetry System," IEEE Geoscience and Remote Sensing Letters, 14, 759-763, 2017.

[2] Bertrand de Saint-Jean, "Étude et développement d'un système de gravimétrie mobile." Géophysique [physics.geo-ph]. Observatoire de Paris, 2008.

[3] Van Camp, M., and Vauterin, P., "Tsoft: graphical and interactive software for the analysis of time series and Earth tides" Computers \& Geosciences, 31(5) 631-640, doi: 10.1016/j.cageo.2004.11.015, 2005.

[4] Jekeli, C., "Geodetic Application", in Inertial navigation systems with geodetic applications., de Gruyter, Germany, 2001

[5] Gerlach, C. and R. Dorobantu. "A Testbed for Airborne Inertial Geodesy : Terrestrial Gravimetry Experiment by INS / GPS." 2004. 